\title{
Emerging antibiotic resistance pattern of urinary tract infection (UTI) due to Escherichia Coli (E.Coli).
}

1. MBBS, FCPS (Medicine) Assistant Professor Medicine The University of Faisalabad.

2. MBBS, FCPS (Medicine) Assistant Professor Medicine DHQ, Hospital, Faisalabad.

3. MBBS, FCPS (Medicine) Assistant Professor Medicine KEMU/Mayo Hospital Lahore.

4. MBBS, FCPS (Nephrology)

Assistant Professor Nephrology The University of Faisalabad.

5. MBBS, FCPS (Gastroenterology) Assistant Professor Gastroenterology The University of Faisalabad.

6. MBBS, FCPS (Medicine), FCPS (Rheumatology) Associate Professor Medicine The University of Faisalabad.

7. MBBS

Medical Officer Pediatric

Government Hospital Samnabad, Faisalabad.

Correspondence Address: Dr. Salman Azhar

Department of Medicine The University of Faisalabad salman_azhar2010@yahoo.com

Article received on:

26/01/2021

Accepted for publication: 22/04/2021
Salman Azhar ${ }^{1}$, Talha Munir², Mian Sajjad Ahmed ${ }^{3}$, Wasif Baig ${ }^{4}$, Ahmed Nawaz Baber $^{5}$, Khalid Pervez $^{6}$, Ahsan Jahangir ${ }^{7}$

ABSTRACT... Objective: To determine the emerging antibiotic resistance pattern of adult patients of MDR UTI cases caused by E.Coli. Study Design: Retrospective Data. Setting: Departments of Medicine and Obstetrics \& Gynecology, Madinah Teaching Hospital / University Medical and Dental College, Faisalabad. Period: August 2016 to Sep 2018. Material \& Methods: This retrospective study included 187 patients meeting the operational definition of multi-drug resistance cases of urinary tract infection caused by $\mathrm{E}$. coli. All cases either male or female of age above 15 years with positive reports of urine culture and sensitivity for E.coli were included in the study. Along with the demographic variables urine WBC's, results of urine culture and sensitivity were noted. Antibiotic sensitivity pattern of all the E.coli resistant UTI cases was analyzed. After identification of the microbe, antimicrobial sensitivity was accessed using KirbyBauer disc diffusion method on the Mueller-Hinton agar. The antimicrobial susceptibility test was performed against E. coli strains by using the following antibiotics: Piperacilin, AmoxicilinCalvulanic, Pipmedic Acid, Cefipime, Ceftrixone, Cefuroxime, Cefoperazone-sulbactam, Ceftazidime, Cephradine, Ciprofloxacin, Ofloxacin, Levofloxacin, ofloxacin, Nitrofurantoin, Amikacin, Tombramycin, Gentamicin, Linezolid, Doxycycline, Sulphamethoxazole, Imipenem, Meropenem, Aztreonam in order to obtain an antibiogram. Results: In our data, 66.8\% $(n=125)$ were MDR UTI, and more than half of these cases were $63.2 \%(n=79)$ were females. Among the total 187 cases, $97.3 \%$ patients were resistant to most of the beta-lactam antibiotics, $95.7 \%$ were resistant to most of the quinolones and $68.4 \%$ were resistant to most of the aminoglycosides. Conclusion: This increasing antibiotic resistance is very alarming and steps should be taken to reduce the misuse of antibiotics. Studies should be done to control the risk factors leading to E. coli UTI. By following proper pharmacological guidelines, principles to use antibiotics and manage outpatient cases of UTIs, the incidence of MDR UTI can be controlled.

Key words: $\quad$ Cystitis, E.Coli, Gram Negative, MDR, Sensitivity, UTI, Urinary Infection.

Article Citation: Azhar S, Munir T, Ahmed MS, Baig W, Baber AN, Pervez K, Jahangir A. Emerging antibiotic resistance pattern of urinary tract infection (UTI) due to Escherichia Coli (E.Coli). Professional Med J 2021; 28(12):1804-1811. https://doi.org/10.29309/TPMJ/2021.28.12.6364

\section{INTRODUCTION}

Infections of the renal tract including kidneys, ureter and bladder affect humans of all age groups. Around one fourth of all the patients presenting with some sort of infection in the hospitals have urinary tract infection (UTI). ${ }^{1}$ Bacteriuria with or without any complaints regarding renal system or with vague symptoms can still be labeled as UTI. The condition, Bacteriuria can be defined as the presence of more than $10^{5}$ colony forming units (CFU) in one $\mathrm{ml}$ urine sample. ${ }^{2}$

A study reported that globally about a million patients with UTI symptoms visit emergency on a daily basis; with a large number of hospital admissions as well. These symptoms include burning sensation with passage of urine, frequent urge to pass urine, lower abdominal pain or pain in the back, sometimes urine becomes cloudy or darker than normal and even smelly along with generalized malaise and tiredness. . $^{3,4}$

Normal flora apart from the GI tract can also reside in the urethra. These include S. epidermidis, S. faecalis and Corynebacterium. With urinary tract infection, microbes climb up the renal tract from urethra to bladder and start to grow. Most of these microbes are fecal borne. More than three fourth 
of these infections are caused by a fecal borne microbe namely E.coli. The earlier we get rid of this infection from the urinary tract the lesser the morbidity can be and complications can be avoided. $^{5}$

Multiple antimicrobials are used to treat E. coli UTI cases. These include nitrofurantoin or trimethoprim-sulfamethoxazole for uncomplicated cases whereas quinolones, ceftriaxone, aminoglycosides and carbapenems for infections in ureter, kidneys and multiple parts of urinary tract. With the miss-use of antibiotics, these is a rise in the multidrug-resistant (MDR) cases of Enterobacteriaceae UTI. Every population has its own pattern of antibiotic resistance. ${ }^{6}$

A local study conducted in Multan, reported MDR E. coli isolates resistant pattern of $80 \%$ (ImipenemIMI), 72\% (Ciprofloxacin-Cip), 68\% (AugmentinAug), 60\% (Cefixime-Cfm), and 52\% (Gentamicin$\mathrm{CN})$, respectively, while these microbes were sensitive to (piperacillin/tazobactum-TZP) 70\%, (Amikacin-AK) 62\% and $F(60 \%)$.

We collected data and reported the demographic variables and prevalence of MDR E. coli UTI cases of our local population, Faisalabad, Punjab, Pakistan. In this second phase, antimicrobial sensitivity pattern was analyzed for these cases. Primary objective of this study was to determine the emerging antibiotic resistance pattern of MDR UTI cases caused by E.Coli at Madinah Teaching Hospital and Maqsooda Zia Hospital, Faisalabad.

\section{MATERIAL \& METHODS}

This retrospective study included 187 patients meeting the operational definition of multi-drug resistance cases of urinary tract infection caused by $E$. coli. The objective of this study was to determine the antibiotic resistance pattern of these drug resistant cases of E. coli UTI. This was conducted in the department of Medicine, Madinah Teaching Hospital and Maqsooda Zia Hospital, Faisalabad. Electronic record from the department of medicine and their labs from the pathology department was analyzed. All cases either male or female of age above 15 years with positive reports of urine culture and sensitivity for
E.coli were included in the study.

MDR E.coli UTI cases were those who showed resistance to 2 or more of the three antimicrobial groups: (1) Beta-lactames (2) Quinolones (3) Aminoglycosides. Age, gender, urine WBC's and results of urine culture and sensitivity were noted on a designed proforma.

After approval form the hospitals' ethical committee (TUF/Dean/2016/45), electronic medical records of outdoor, indoor and emergency department patients (from medicine, urology, gynecology and surgery) were used to fill out our case proformas. Age of the patients was stratified into young adults ( $<35$ years), adults (36 to 60 years), and elderly (61 to 90 years).

Along with the demographic variables urine WBC's, results of urine culture and sensitivity was noted. Antibiotic sensitivity pattern of all the E.coli resistant UTI cases was analyzed. After identification of the microbe, antimicrobial sensitivity was accessed using Kirby-Bauer disc diffusion method on the Mueller-Hinton agar. The antimicrobial susceptibility test was performed against $\mathrm{E}$. coli strains by using the following antibiotics: Piperacilin, AmoxicilinCalvulanic, Pipmedic Acid, Cefipime, Ceftrixone, Cefuroxime, Cefoperazone-sulbactam, Ceftazidime, Cephradine, Ciprofloxacin, Ofloxacin, Levofloxacin, ofloxacin, Nitrofurantoin, Amikacin, Tombramycin, Gentamicin, Linezolid, Doxycycline, Sulphamethoxazole, Imipenem, Meropenem, Aztreonam in order to obtain an antibiogram.

All the variables were analyzed using SPSS 20. All the nominal variables were expressed as percentages and the numerical data was expressed as mean with standard deviation. To control the effect modifiers, data was stratified for age, gender and post-stratification chi square was applied to see the effect on the outcome. P-value of $<0.05$ was taken significant.

\section{RESULTS}

The data collected in this study included 187 UTI patients with $\mathrm{E}$. coli being the causative agent. 
Data regarding the sensitivity pattern of these positive cultures of $\mathrm{E}$. coli was documented. Among the E. coli UTI patients included in the study using non-probable consecutive sampling technique, around $2 / 3^{\text {rd }}$ were females. Majority cases were of adult group from 35 to 60 years age.

Table-I shows the basic data of all the patients included in the study. As all these cases were diagnosed after urine complete test for pus on microscopy, all showed some amount of pus. A detail regarding the pus on microscopy is shown in the Table-I. We categorized the number of pus cells seen on a high power field of microscope (HPF) into three: presence of $3-20$ pus cells as 'few'; 20 to 40 pus cells per HPF a numerous and presence of $>40$ pus cells was labeled as field full.

In our data, $66.8 \%(n=125)$ were MDR UTI, and more than half of these cases were $63.2 \%(n=79)$ were females. Anti-microbial sensitivity pattern was noted for each case and the percentage of patients resistant and sensitive to various anti- microbials were noted, as shown in the TableII. Among the total 187 cases, 97.3\% patients were resistant to most of the beta-lactam antibiotics, $95.7 \%$ were resistant to most of the quinolones and $68.4 \%$ were resistant to most of the aminoglycosides. Figures and tables below shows the details of anti-microbial sensitivity pattern of E. coli UTI.

\begin{tabular}{|l|c|c|c|}
\hline \multicolumn{2}{|c|}{ Variables } & Frequency & $\begin{array}{c}\text { Percent } \\
\%\end{array}$ \\
\hline Age & Young adult & 39 & 20.9 \\
\hline & Adult & 97 & 51.9 \\
\hline Gender & Elders & 51 & 27.3 \\
\hline & Male & 71 & 38.0 \\
\hline Pus on & Female & 116 & 62.0 \\
\hline microscopy & Numerous & 36 & 19.3 \\
\hline & $20-40$ & 96 & 51.3 \\
\hline & Field Full $>40$ & 54 & 28.9 \\
\hline
\end{tabular}

Table-I. Showing the details of patients regarding age, gender and pus in urine.

\section{$\%$ of E. coli UTI cases Resistant to anti-microbials}

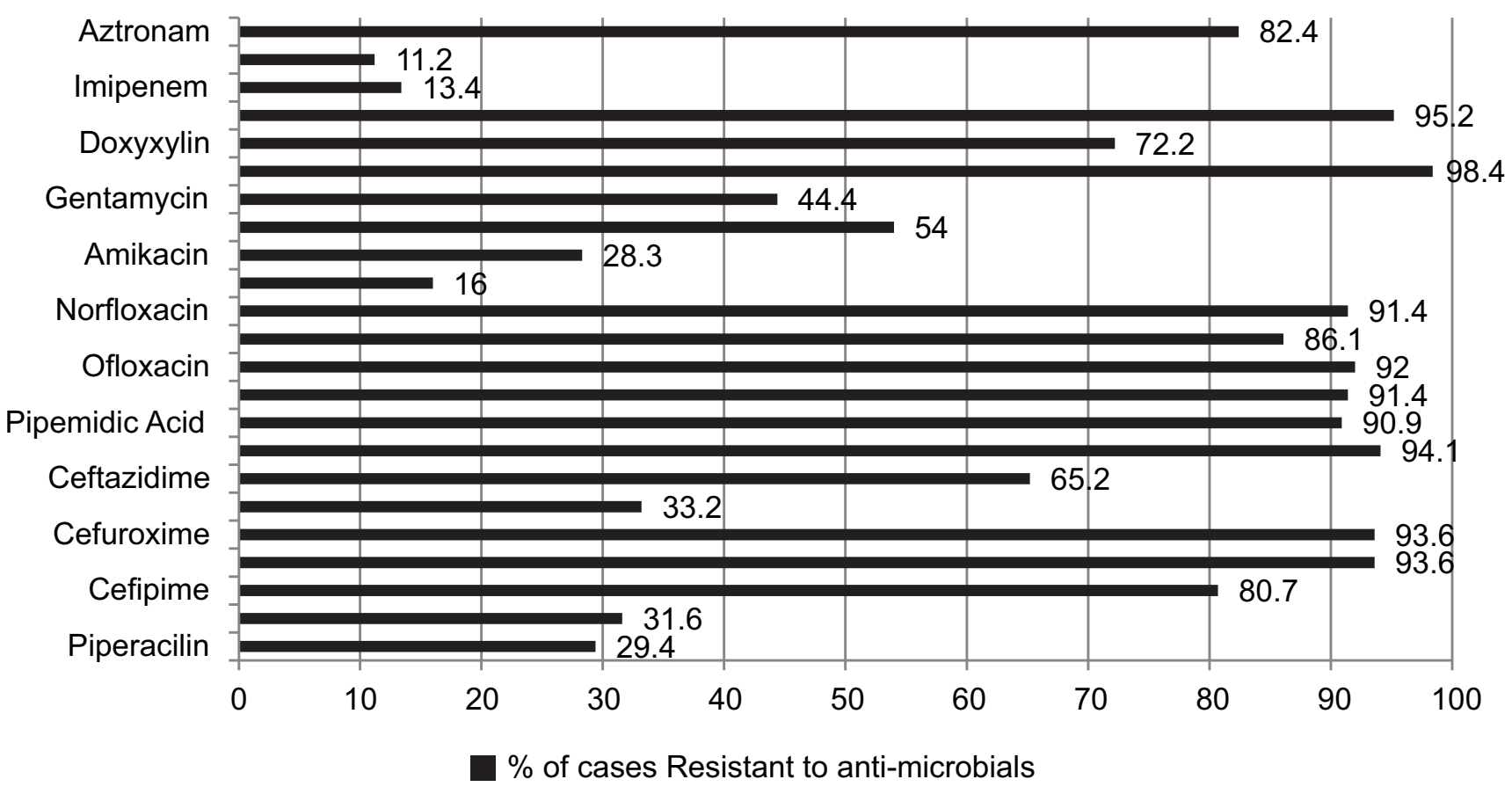

Figure-1. Showing percentage of patients resistant to various anti-microbial as part of sensitivity pattern. 
$\%$ of E. coli UTI cases sensitive to anti-microbials

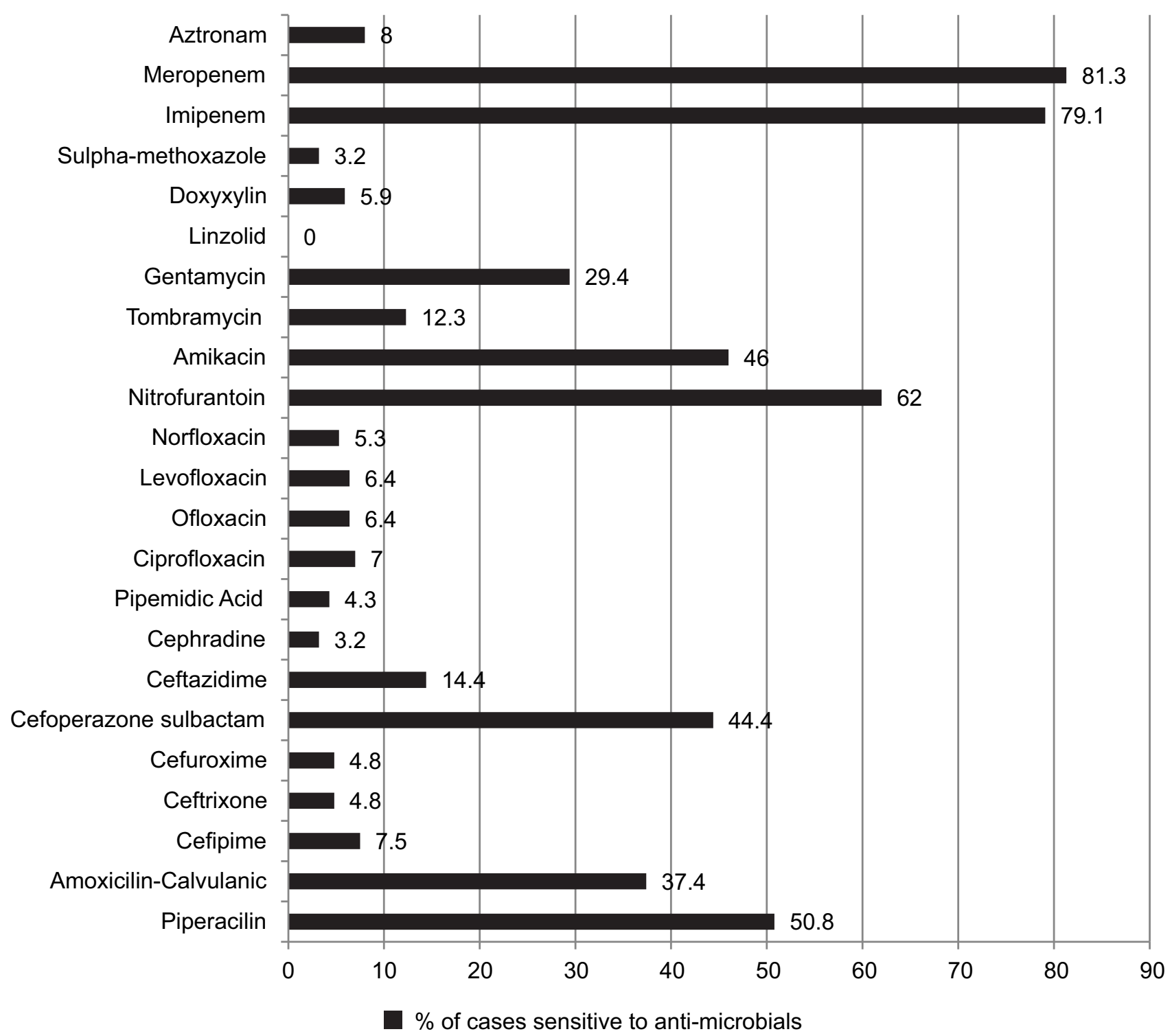

Figure-2. Showing the percentage of patients sensitive to various anti-microbials as part of sensitivity pattern.

Frequency of multi-drug resistant (MDR) E. coliUTI cases compared among male and female gender and among various age groups. Among all these cases, more than $70 \%$ of patients were resistant to ceftazidime, cephradine, pipemidic acid, ciprofloxacin, ofloxacin, levofloxacin, norfloxacin, linezolid, doxycyline and sulpha-methoxazole. While around $50 \%$ cases were sensitive to only piperacillin, cefoperazone, nitrofurantoin and amikacin.
Among the total 125 of 187 (66.8\%) patients diagnosed as MDR E. coli UTI, 63.2\% $(n=79)$ were female and $36.8 \%$ ( $n=46$ of 125 ) were male $(p=0.29)$. Taking into account the 3 major groups of anti-microbial, aminoglycosides, betalactams and quinolones, almost all of the MDR E. coli cases were resistant to one or more drugs in all the three major types of anti-microbial. 


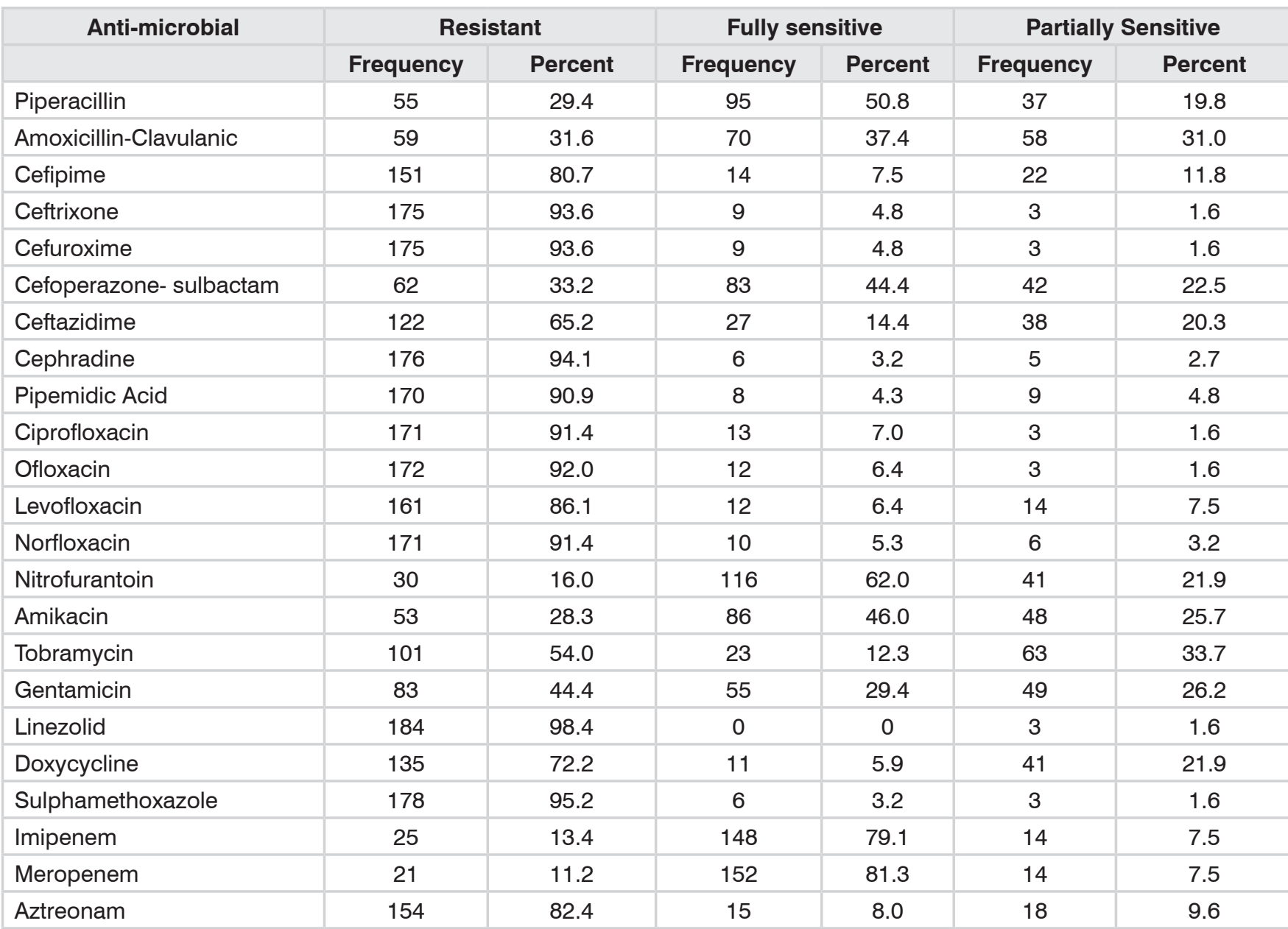

Table-II. Showing the detailed percentage of patients resistant to, sensitive to and partially sensitive to various antimicrobial.

\section{DISCUSSION}

Urinary tract infections are commonly seen in all age groups and in both genders. Children and old age group people are more affected and female gender is a significant risk factor. It is also one of the most common hospital acquired infection patients can get when admitted in the wards with other diagnosis. As antimicrobial resistance of E. coli is rising, it becomes very difficult for the consultants to select an appropriate oral antimicrobial. $^{7}$

Phase I of this study was published showing the demographic variables and prevalence of MDR E. coli UTI cases of Faisalabad, Punjab, Pakistan. This is the second phase of this study in which antimicrobial sensitivity pattern was analyzed. To our knowledge, the prevalence of E. coli UTI among the population of Faisalabad has been first time documented in this study. Analysis of data was done to know the various risk factors, distribution of E. coli UTI cases with regard to various age groups and gender-most commonly affected and gender-wise distribution. All the cases of our study were of local population and were of different socioeconomic status. Most of the cases were poor and belonged to poor socioeconomic status with poor quality of drinking water and sanitary conditions as around half of the local population.

A study done in Nepal, South Asia analyzed the urine samples of patients of UTI and reported that microbes were seen in $1 / 4^{\text {th }}$ samples, majority of which were of female gender $(60 \%)$; this female prevalence of culture positive UTI cases was also seen in our study. This study also reported that more than $50 \%$ of these cases were caused by $\mathrm{E}$. 
coli. ${ }^{8}$ In their study, around half of the microbes were sensitive to gentamicin (57\%), ceftriaxone (51\%); unlike that seen in our population of culture positive UTI cases and nitrofurantoin (45.75\%), and least sensitivity was seen for ampicillin $(5.3 \%)$; this was $62 \%$ for nitrofurantoin and least sensitivity was for linezolid.

A study included most advanced aged cases of MDR UTI cases, McAllister $\mathrm{R}$, et al. reported that around $15 \%$ of the old age patients (>80 years) had asymptomatic pyuria. They concluded that advanced age, genital or urinary tract anatomical anomalies, those with history of renal stones, bladder stones, and recurrent episodes of dehydration and diabetic patients are more prone to develop urinary tract infections. These were also the risk factors of development of multi-drug resistance in UTI. ${ }^{9}$

In a local study done in a tertiary hospital in Multan, 150 samples with E. coli MRD UTI, and antimicrobial susceptibility were tested. Demographic analysis showed that patients of rural background were more affected, with female predominantly involved. Resistance was seen against imipenem, Ciprofloxacin, Augmentin as $80 \%, 72 \%$ and $68 \%$, respectively. ${ }^{10}$ Our study shows similar sensitivity pattern for ciprofloxacin and augmentin but still E. coli were sensitive to imipenem.

It was observed that, in each and every age group the percentage of female patients were higher than male. The percentage of male and female patients was highest in the age group between $21-30$ years which was $7 \%$ and $28.90 \%$ respectively. The lowest percentage of male and female patients was in the age group $<10$ years which was $2.10 \%$ and $2.80 \%$ respectively. The present data stated that, the onset of UTIs in female were high at the age between 2130 years (28.90\%). Amoxicillin, Doxycycline, Cephalexin, Cephradine, Co-trimoxazole, Cefixime, Ceftriaxone and Ciprofloxacin showed less sensitivity to E. coli. But Cefuroxime showed significant (mean p-value $<0.001$ ) level of sensitivity to $E$. coli. ${ }^{11}$

A meta-analysis of around 25 studies with MDR
UTI patients showed that major factor leading to MDR UTI was the miss use of antibiotics. This reported in 16 out of 20 studies. Other significant factors were catheterization and history of hospital stay. $^{12}$

Another study reported after analyzing cases of UTI caused by E. coli and concluded that females were mostly involved and resistance was most commonly seen against trimethoprimsulfamethoxazole, ampicillin and ampicillinsulbactam. Resistance against other antibiotics was ciprofloxacin (47.3\%), levofloxacin (43.6\%) and cephalosporins (27.6\%); this was much different in our population, as our population was almost completely resistant to these antimicrobials. MDR UTI E. coli cases were around reported $63 \%$, as that in our population. Most of cases in this group with resistance to fluoroquinolone (FQ) were MDR cases and were of male gender. Those with history of hospital stay were resistant to $3^{\text {rd }}$ gen cephalosporin and nitrofurantoin. ${ }^{13}$ Our data was limited and we couldn't document history of hospital stay and catheterization as variables in our study.

Unlike that seen in our study population, Linsenmeyer K, et al. evaluated the data of 126 cases of Gram negative MDR UTI, reported the mean age of 72.8 years and $90 \%$ male gender. ${ }^{14}$ In another study in which more than 200 cases were studied to identify MDR E. coli UTI cases, the frequency of multi-drug resistant cases was reported to be $54.5 \%$ (121 patients were diagnosed with $\mathrm{E}$. coli and multi-drug resistance, out of the 222 total E. coli UTI cases). ${ }^{15}$ This was $62 \%$ in our study.

A large group of UTI cases were studied in a study, in which Ukah UV, et al reports $1238 \mathrm{E}$. coli UTI patients. Around $40 \%$ patients were resistant to single group of antimicrobial and $25 \%$ patients were MDR, unlike the results of our study. Analysis showed that factors like age, education, economic status showed no association with the frequency of MDR UTI cases. A significant risk factor was the frequent use of antibiotics in the last 6 months; ${ }^{16}$ this factor was not documented in our study. 
Elsayed TI, et al. collected data of resistant cases of UTIs with history of recurrent episode and tested them for antibiotic resistance. They reported that resistance was seen against ampicillin in 95\% cases of MDR UTI, Sulphamethoxazole/ Trimethoprim 69\%, Nalidixic acid 70\%, Norfloxacin 59\%, Gentamicin 31\%, Nitrofurantoin $16 \%$, Cephalothin $93 \%$ and Imipenem $2 \%$. They reported that among all the MDR culture positive UTI cases around $95 \%$ were caused by E. coli. They also reported that almost all the MDR cases were Extended Spectrum Beta-Lactamase (ESBL) producers. ${ }^{17}$

A large study done in Europe included around 30 countries and collected data regarding multidrug-resistant cases of infections involving the urinary tract. E. coli and K. pneumonia infections were the most prevalent pathogens in MDR cases in most of the countries. Resistance was seen to penicillin (65\%), aminoglycosides (19\%) and quinolones (44\%) among all the E. coli cases. Our study reported much more than that reported in this study. With every crossing year, the number of cases of ESBL-positive E. coli infections also increased all around in the European Union $(\mathrm{EU}){ }^{18}$

\section{CONCLUSION}

MDR UTIs can occur as secondary infections leading to rise in morbidity and hospital stay. Bottom-line of our discussion is that the most of the MDR UTI cases are resistant to betalactam agents, trimethoprim-sulfamethoxazole, fluoroquinolones and aminoglycosides. Fosfomycin and nitrofurantoin are seen clinically efficient, but resistance against these agents is also increasing. Our local population have shown much worse sensitivity pattern toward various antimicrobial agents, due to excessive misuse. This increasing antibiotic resistance is very alarming and steps should be taken to reduce the misuse of antibiotics. Studies should be done to control the risk factors leading to E. coli UTI. By following proper pharmacological guidelines, principles to use antibiotics and manage outpatient cases of UTIs, the incidence of MDR UTI can be controlled. Copyright@ 22 Apr, 2021.

\section{REFERENCES}

1. Badr AA, Shaikh GA. Recurrent urinary tract infections management in women. Sultan Qaboos Univ Med J. 2013 Aug; 13(3): 359-367.

2. Soto DSM. Treatment of UTI according to age and gender. Barcelona institute for global health. [internet]. 2015 [cited Dec 2018]; [about $34^{\circ} \mathrm{p}$ ]. Available from: http://www.escmid.org/escmid_publications/escmid_ elibrary $/ \mathrm{material} / ? \mathrm{mid}=29443$.

3. Badalato G, Kaufmann M. Medical student curriculum: Adult UTI. American Urology Association [internet]. 2016 [cited Dec 2018]; [about $10^{\circ} \mathrm{p}$ ]. Available from: http://www.auanet.org/education/auauniversity/formedical-students/medical-student-curriculum/adult-uti.

4. John AS, Mboto $\mathrm{Cl}$, Agbo B. A review on the prevalence and predisposing factors responsible for urinary tract infection among adults. European Journal of Experimental Biology. 2016; 6(4): 7-11.

5. Ayub M, Amir JS, Firdous K, et al. E. coli. The most prevalent causative agent urinary tract infection in pregnancy: Comparative analysis of susceptibility and resistance pattern of antimicrobials. Arch Clin Microbiol. 2016, 7:4.

6. Sanchez GV, Master RN, Karlowsky JA, Bordon JM, "In vitro antimicrobial resistance of urinary Escherichia coli isolates among U.S. outpatients from 2000 to 2010," Antimicrobial Agents and Chemotherapy, 2012: 56(4);2181-83.

7. Walker E, Lyman A, Gupta K, Mahoney MV, Snyder GM, Hirsch EB. Clinical management of an increasing threat: Outpatient urinary tract infections due to multidrug-resistant uropathogens. Clin Infect Dis. 2016; 63(7):960-965. doi:10.1093/cid/ciw396.

8. Awasthi TR,Pant ND, Dahal PR. Prevalence of multidrug resistant bacteria in causing community acquired urinary tract infection among the patients attending outpatient department of seti zonal hospital, dhangadi, Nepal. Nepal Journal of Biotechnol. 2015; 3(1):55-9.

9. McAllister R, Allwood J. Recurrent multidrug resistant urinary tract infections in geriatric patients Fed Pract. 2014; $31(7): 32-5$.

10. Fatima T, Rafiq S, Iqbal A. Prevalence and Antibiogram of MDR E. coli Strains Isolated from UTI Patients-1Year Retrospective Study at Nishtar Medical Hospital, Multan. SN Compr. Clin. Med. 2020; 2, 423-431. 
11. Shahina Z, Islam J, Abedin J, Chowdhury I, et al. A study of antibacterial susceptibility and resistance pattern of $\mathrm{E}$. coli causing urinary tract infection in Chittagong, Bangladesh. Asian Journal of Biological Sciences, 2011; 4: 548-555.

12. Tenney J, Hudson N, Alnifaidy H, Cheung Li JT, Fung $\mathrm{KH}$. Risk factors for acquiring multidrug-resistant organisms in urinary tract infections. A systematic literature review Saudi Pharma J. 2018; 26:678-84.

13. Castillo FR, Moreno F, Avelar FJ, Márquez F, Harel J, Guerrero AL, et al. An evaluation of multidrug-resistant Escherichia coli isolates in urinary tract infections from Aguascalientes, Mexico: Cross-sectional study. Ann Clin Microbiol Antimicrob 2018; 17:1-34.

14. Linsenmeyer K, Strymish J, Gupta K. Two simple rules for improving the accuracy of empiric treatment of multidrug-resistant urinary tract infections. Antimicrob Agents Chemother. 2015; 59(12):7593-7596. doi:10.1128/AAC.01638-15.
15. Mutters NT, Mampel A, Kropidlowski R, Biehler K, Günther $\mathrm{F}$, Balu I, et al. Treating urinary tract infections due to MDR E. coli with Isothiocyanates a phytotherapeutic alternative to antibiotics Fitoterapia 2018;129:237-40.

16. Ukah UV, Glass M, Avery B, Daiganault D, Mulvey MR, Reid RJ, et al. Risk factors for acquisition of multidrugresistant escherichia coli and development of community-acquired urinary tract infections Epidemiol. Infect. 2018; 146:46-57.

17. Elsayed TI, Ismail AF, Elgamal SA, Gad AH. The occurrence of multidrug resistant e. coli which produce esbl and cause urinary tract infections. J Appl Microbiol Biochem. 2017; 1(2):8.

18. Mazzaroiol A, Bazaj A, Cornaglia G. Multi-drugresistant gram-negative bacteria causing urinary tract infections: A review Journal of Chemotherapy 2017; 29:2-9.

\begin{tabular}{|c|c|c|c|}
\hline \multicolumn{4}{|c|}{ AUTHORSHIP AND CONTRIBUTION DECLARATION } \\
\hline No. & Author(s) Full Name & Contribution to the paper & Author(s) Signature \\
\hline 1 & $\begin{array}{l}\text { Salman Azhar } \\
\text { Talha Munir }\end{array}$ & $\begin{array}{l}\text { Concept, Paper writing, data } \\
\text { collection. } \\
\text { Statistical analysis. }\end{array}$ & \\
\hline 3 & Mian Sajjad Ahmed & Manuscript revision. & \\
\hline 4 & Wasif Baig & Discussion, Conclusion. & intan ... \\
\hline 5 & Ahmed Nawaz Baber & Data collection. & \\
\hline 6 & Khalid Pervez & Paper writing. & $i k$ \\
\hline 7 & Ahsan Jahangir & $\begin{array}{l}\text { Paper writing, Statistical } \\
\text { analysis. }\end{array}$ & \\
\hline
\end{tabular}

